\title{
Characteristics of Tuberculosis in Patients with Rheumatoid Arthritis: A Retrospective Single-center Study
}

\author{
Takashi Ishiguro, Noboru Takayanagi, Naho Kagiyama, \\ Tsutomu Yanagisawa and Yutaka Sugita
}

\begin{abstract}
Objective The objective of this study was to assess the characteristics of hospitalized patients suffering from tuberculosis with rheumatoid arthritis (RA), focusing specifically on those treated without anti-tumor necrosis factor (TNF) therapy.

Methods We conducted a retrospective review to assess the characteristics of 1,022 hospitalized Japanese patients suffering from tuberculosis with and without RA between 2002 and 2011.

Results Compared with the 995 non-RA patients with tuberculosis, the 24 RA patients with tuberculosis treated without anti-TNF therapy were older and predominantly female. They less frequently had a history of smoking, with a higher rate of underlying respiratory diseases, an impaired performance status and side effects. The three RA patients receiving anti-TNF therapy had extrapulmonary tuberculosis; however, the rate of extrapulmonary tuberculosis in the other RA patients treated without anti-TNF therapy did not differ from that observed in the non-RA patients. Five patients died during hospitalization. The in-hospital mortality of the RA patients did not differ significantly from that of the non-RA patients. Independent factors contributing to in-hospital mortality did not include RA.

Conclusion In this study, the RA patients treated without anti-TNF therapy did not differ from the non-RA patients in terms of the rate of extrapulmonary tuberculosis or bilateral pulmonary lesions, although they did exhibit a higher frequency of side effects of antituberculosis treatment. RA was not found to be an independent factor contributing to in-hospital mortality.
\end{abstract}

Key words: tuberculosis, rheumatoid arthritis, characteristics, biologics, tumor necrosis factor

(Intern Med 53: 1291-1298, 2014)

(DOI: 10.2169/internalmedicine.53.1936)

\section{Introduction}

Rheumatoid arthritis (RA) is an autoimmune disease that results in chronic systemic inflammation affecting many tissues and organs. Over the past 10 years, the clinical course of patients with active RA not controllable with conventional anti-rheumatic drugs has been modified, with good results, due to the introduction of a new class of anti-cytokine drugs, including anti-tumor necrosis factor (TNF) therapy, thus altering the entire treatment strategy for RA. However, problems of increased rates of infection, including tuberculosis, in RA patients have emerged.

Patients undergoing anti-TNF therapy frequently develop extrapulmonary tuberculosis (1-4). However, it remains unknown whether this high frequency of extrapulmonary tuberculosis is a characteristic of RA patients undergoing antiTNF therapy or whether anti-TNF therapy is not a factor in RA patients, as the characteristics of tuberculosis infection in RA patients treated without anti-TNF therapy have not been fully elucidated.

RA is generally considered to be associated with an increased risk of tuberculosis. In Japan, the prevalence of RA among all tuberculosis patients is reported to be approximately three times as high as that of RA in the entire population (5), and another report showed an increased risk of 3.21 for developing tuberculosis in Japanese patients with RA (6) compared with the general population. An increased 
relative risk of tuberculosis in patients with RA has also been reported in Sweden (7) and Spain $(8,9)$.

In order to assess the characteristics of tuberculosis in RA patients undergoing anti-TNF therapy, the characteristics of tuberculosis in RA patients treated without anti-TNF therapy should be elucidated. Therefore, the aim of the present study was to clarify the characteristics of tuberculosis in patients with RA, with a special focus on the characteristics of the disease in those treated without anti-TNF therapy.

\section{Materials and Methods}

We retrospectively studied all patients hospitalized with tuberculosis at our institution in Saitama, Japan, between January 2002 and December 2011. The study protocol was approved by the Ethics Committee of Saitama Cardiovascular and Respiratory Center. Tuberculosis was diagnosed based on the presence of positive culture results for Mycobacterium tuberculosis, and RA was diagnosed according to established classification criteria (10) by rheumatologists certified by the Japan College of Rheumatology. The performance status (PS) was judged according to the Eastern Cooperative Oncology Group criteria (11), and the chest Xray findings on admission were judged based on the classification of the Japanese Society for Tuberculosis guidelines (12) instituted by the tuberculosis advisory committee according to national law concerning the prevention of infections and medical care for patients suffering from infectious disease in Japan.

Acid-fast staining was performed using fluorochrome staining with phenolic auramine-rhodamine. The culture methods used to assess $M$. tuberculosis included commercial automated mycobacterial growth indicator tube systems, the BACTEC 960 TB system (MIGIT, Becton Dickinson Microbiology Systems, Sparks, USA) or 2\% Ogawa solid medium (Japan BCG Laboratory, Tokyo, Japan). The identification of M. tuberculosis was performed based on the methods of DNA-DNA hybridization (DDH Mycobacteria ${ }^{\circledR}$, Kyokuto Pharmaceutical Co., Ltd., Tokyo, Japan), immunochromatography (Capilia $\mathrm{TB}^{\circledR}$, Tauns, Kamishima, Japan) and polymerase chain reaction.

The antituberculosis treatment regimens were planned by physicians at our institution according to established guidelines $(12,13)$. Six-month regimens included isoniazid (INH), rifampin (RIF), pyrazinamide (PZA), ethambutol (EMB) or streptomycin (SM) (four drugs for two months, followed by INH + RIF for four months). Nine-month regimens included INH, RIF, EMB or SM (three drugs for the initial two months, followed by INH + RIF for seven months). Other treatment regimens were defined as 'others.' In patients receiving corticosteroids or immunosuppressants, including disease-modifying antirheumatic drugs (DMARDs), the treatment period was prolonged for three months.

The treatment strategies with antirheumatic drugs were planned by the rheumatologists who introduced each patient to our hospital, and the antirheumatic drugs administered immediately prior to the development of tuberculosis, during hospitalization for antituberculosis treatment and at the latest contact point with the patient were recorded.

The patients were discharged from the hospital when the following criteria determined according to the national law concerning the prevention of infections and medical care for patients suffering from infectious disease were met: 1) the disappearance of symptoms, including cough and fever, in addition to negative culture results of sputum samples obtained over another three days or 2) negative results of the smear or culture of sputum samples obtained over another three days after two weeks from the initiation of standard antituberculosis treatment.

The antituberculosis therapy was considered complete when the completion criteria defined according to the guidelines (13) were met. Relapse was defined as the occurrence of culture-positive findings after the completion of therapy in patients who achieved culture-negative findings under treatment with antituberculosis drugs. The cause of death was that listed on the certificate of death.

\section{Statistical analysis}

The results are presented as numbers and percentages or the mean $\pm \mathrm{SD}$, unless otherwise indicated. Student's $t$-test, the Wilcoxon rank-sum test and the chi-square test with Fisher's exact test were used for the statistical analysis. Risk factors for in-hospital mortality due to RA were evaluated using univariate and multivariate logistic regression analyses. Variables exhibiting significance in the univariate analysis ( $p$ $<0.05$ ) were included in the multivariate logistic regression analysis using the backward elimination method. The $95 \%$ confidence intervals (CIs) were also reported for all comparisons. In all instances, a two-tailed $\mathrm{p}$ value of $<0.05$ was considered to indicate a statistically significant result. All statistical analyses were performed using the Statistical Analysis System (SAS) software program version 9.1.3 (SAS Institute, Inc., Cary, USA).

\section{Results}

From 2002 to $2011,1,022$ patients with tuberculosis were hospitalized. In total, 27 (2.6\%) of these patients had RA, with a median disease duration of 9.5 years (range, 1-62 years). Three of these 27 patients received anti-TNF therapy after developing tuberculosis.

\section{Characteristics of the RA patients treated without anti-TNF therapy}

\section{Demographics}

The RA patients treated without anti-TNF therapy were predominantly women; nine $(37.5 \%)$ of the 24 patients were men, whereas $680(68.3 \%)$ of the 995 non-RA patients were men, a significant difference between the RA and non-RA patients (Table 1). The underlying diseases in the RA pa- 
Table 1. Characteristics of Patients with RA without Anti-TNF Therapy and Patients without RA

\begin{tabular}{|c|c|c|c|c|c|}
\hline & \multicolumn{2}{|c|}{$\begin{array}{l}\text { RA patients } \\
(\mathrm{n}=24)^{*}\end{array}$} & \multicolumn{2}{|c|}{$\begin{array}{l}\text { Non-RA patients } \\
\quad(\mathrm{n}=995)\end{array}$} & \multirow[t]{2}{*}{$\mathrm{p}$ value } \\
\hline & $\mathrm{n}$ & $(\%)$ & $\mathrm{n}$ & $(\%)$ & \\
\hline \multicolumn{6}{|c|}{ Duration from initial symptoms to admission } \\
\hline$<1$ month & 13 & $(54.2)$ & 345 & $(34.7)$ & \\
\hline$\geq 1$ month & 0 & $(0)$ & 463 & $(46.5)$ & $<0.001$ \\
\hline Unknown & 11 & $(45.8)$ & 187 & $(18.8)$ & Excluded \\
\hline Male sex & 9 & $(37.5)$ & 680 & $(68.3)$ & 0.003 \\
\hline Age & $74.5 \pm 12.3$ & & $64.3 \pm 20.5$ & & $<0.001$ \\
\hline Smoking history, yes & 6 & $(25.0)$ & 556 & $(55.9)$ & 0.003 \\
\hline \multicolumn{6}{|l|}{ Underlying diseases } \\
\hline Respiratory diseases & 7 & $(29.2)$ & 114 & $(11.5)$ & 0.017 \\
\hline Systemic diseases other than RA & 16 & $(66.7)$ & 531 & $(53.4)$ & 0.219 \\
\hline Extrapulmonary tuberculosis & 6 & $(25.0)$ & 209 & $(21.0)$ & 0.615 \\
\hline \multicolumn{6}{|l|}{ Antirheumatic drugs } \\
\hline Corticosteroids & 20 & $(83.3)$ & 19 & $(1.9)$ & $<0.001$ \\
\hline MTX & 3 & $(12.5)$ & 0 & $(0)$ & $<0.001$ \\
\hline Biologics & 3 & $(11.1)$ & 0 & $(0)$ & $<0.001$ \\
\hline DMARDs other than MTX & 11 & $(45.8)$ & 3 & $(0.3)$ & $<0.001$ \\
\hline NSAIDs & 14 & $(58.3)$ & 0 & $(0)$ & $<0.001$ \\
\hline Symptoms, yes & 22 & $(91.7)$ & 851 & $(85.5)$ & 0.2615 \\
\hline Performance status 3 or 4 & 13 & $(54.2)$ & 253 & $(25.4)$ & 0.004 \\
\hline Respiratory failure, yes & 7 & $(29.2)$ & 168 & $(16.9)$ & 0.166 \\
\hline Positive smear result, yes & 20 & $(83.3)$ & 867 & $(87.1)$ & 0.533 \\
\hline Withdrawal due to side effects, yes & 6 & $(25.0)$ & 68 & $(6.8)$ & 0.006 \\
\hline Change of treatment regimen & 4 & $(16.7))$ & 66 & $(6.6)$ & 0.076 \\
\hline Hospitalization (days), overall & $81.0 \pm 47.5$ & & $74.2 \pm 46.9$ & & 0.483 \\
\hline Outcome, death during hospitalization & 5 & $(20.8)$ & 149 & $(15.0)$ & 0.391 \\
\hline
\end{tabular}

tients included respiratory diseases in seven patients and systemic diseases other than RA in 16 patients. Although respiratory diseases were more frequent in the RA patients than in the non-RA patients, the frequency of systemic diseases did not differ significantly between the RA and nonRA patients. Twenty-two of the RA patients had symptoms when diagnosed with tuberculosis, whereas the other two patients did not, although X-ray examinations performed regularly during patient follow-up detected abnormal lung shadows in these two patients. The patients with RA included 13 patients $(54.2 \%)$ with an impaired PS (PS 3 or 4), which was more frequently seen in the RA patients than in the non-RA patients. Seven of the 24 RA patients had respiratory failure, the frequency of which did not differ significantly between the RA and non-RA patients.

\section{Antirheumatic drugs following the diagnosis of tu- berculosis in the RA patients}

The antirheumatic drugs administered in the RA patients following the development of tuberculosis included corticosteroids in 20 cases $(83.3 \%$, daily prednisolone dose: 6.2 $\pm 2.4 \mathrm{mg}$ ), methotrexate (MTX) in three cases (12.5\%, weekly dose: $5.7 \pm 5.2 \mathrm{mg}$ ), DMARDs other than MTX in 11 cases $(45.8 \%$, one patient received two drugs) and nonsteroidal anti-inflammatory drugs (NSAIDs) in 14 cases $(58.3 \%)$.

\section{Laboratory findings on admission}

The laboratory findings on admission showed lower he- moglobin levels, fewer lymphocytes, higher erythrocyte sedimentation rates, higher lactic dehydrogenase levels, lower total protein and albumin levels and higher C-reactive protein levels in the serum samples in the RA patients than in the non-RA patients (Table 2). The frequency of positive smear results of sputum for acid-fast bacilli did not differ significantly between the RA and non-RA patients.

\section{Radiological findings and extrapulmonary lesions}

The frequency of bilateral lesions on radiological imaging was higher among the RA patients than among the non-RA patients (Table 3). Extrapulmonary lesions were found in six $(25.0 \%)$ of the 24 RA patients, a frequency that did not differ significantly between the RA and non-RA patients (Table 4).

\section{Initial treatment regimens for tuberculosis in the $R A$ patients treated without anti-TNF therapy}

The initial treatment regimens for tuberculosis in the RA patients included INH + RIF + PZA + EMB or SM in nine cases, INH + RIF + EMB or SM in 14 cases and another regimen $(\mathrm{INH}+\mathrm{SM})$ in one case. Side effects were more frequently observed in the RA patients than in the non-RA patients. Ultimately, the treatment regimen for tuberculosis had to be changed in four $(16.7 \%)$ RA patients, due to side effects in three patients (one patient with liver dysfunction, one patient with renal dysfunction and one patient with deafness) and according to the sensitivity test results for antituberculosis drugs in one patient. In contrast, the regimen 
Table 2. Laboratory Findings on Admission

\begin{tabular}{|c|c|c|c|c|}
\hline & & RA patients $(\mathrm{n}=24)^{*}$ & Non-RA patients ( $\mathrm{n}=995)$ & $\mathrm{p}$ value \\
\hline WBC & $\times 10^{3} / \mathrm{mm}^{3}$ & $8.3 \pm 3.6$ & $7.3 \pm 2.9$ & 0.233 \\
\hline $\mathrm{N}$ & $\times 10^{3} / \mathrm{mm}^{3}$ & $7.2 \pm 3.6$ & $5.7 \pm 2.6$ & 0.074 \\
\hline Lym & $\times 10^{3} / \mathrm{mm}^{3}$ & $0.7 \pm 0.7$ & $1.1 \pm 0.6$ & $<0.001$ \\
\hline $\mathrm{Hb}$ & $\mathrm{g} / \mathrm{dL}$ & $11.1 \pm 1.9$ & $12.0 \pm 2.1$ & 0.032 \\
\hline PLT & $\times 10^{4} / \mathrm{mm}^{3}$ & $32.2 \pm 12.9$ & $29.3 \pm 11.4$ & 0.190 \\
\hline ESR & $\mathrm{mm} / \mathrm{h}$ & $79.5 \pm 38.3$ & $62.6 \pm 36.6$ & 0.043 \\
\hline AST & $\mathrm{IU} / \mathrm{L}$ & $31.8 \pm 19.6$ & $37.6 \pm 52.9$ & 0.396 \\
\hline ALT & $\mathrm{IU} / \mathrm{L}$ & $24.6 \pm 23.8$ & $27.8 \pm 44.3$ & 0.793 \\
\hline LDH & $\mathrm{IU} / \mathrm{L}$ & $250.0 \pm 79.8$ & $220.5 \pm 99.0$ & 0.005 \\
\hline $\mathrm{ChE}$ & $\mathrm{IU} / \mathrm{L}$ & $153.0 \pm 69.0$ & $206 \pm 308.8$ & 0.111 \\
\hline TP & $g / d L$ & $6.4 \pm 1.0$ & $6.6 \pm 1.1$ & 0.018 \\
\hline Alb & $\mathrm{g} / \mathrm{dL}$ & $2.7 \pm 0.5$ & $3.1 \pm 0.8$ & 0.010 \\
\hline T-cho & $\mathrm{mg} / \mathrm{dL}$ & $172.0 \pm 49.7$ & $154.5 \pm 42.0$ & 0.262 \\
\hline BUN & $\mathrm{mg} / \mathrm{dL}$ & $16.8 \pm 7.4$ & $15.7 \pm 10.1$ & 0.178 \\
\hline Cre & $\mathrm{mg} / \mathrm{dL}$ & $0.7 \pm 0.2$ & $0.8 \pm 0.6$ & 0.469 \\
\hline CRP & $\mathrm{mg} / \mathrm{dL}$ & $7.9 \pm 4.8$ & $6.0 \pm 6.3$ & 0.012 \\
\hline
\end{tabular}

*RA patients with anti-tumor necrosis factor therapy are excluded. RA: rheumatoid arthritis, WBC: white blood cells, PLT: platelets, N: neutrophils: Lym: lymphocytes, AST: aspartate aminotransferase, ALT: alanine aminotransferase, LDH: lactate dehydrogenase, ChE: cholinesterase, TP: total protein, Alb: albumin, T-cho: total cholesterol, BUN: blood urea nitrogen, Cre: creatinine, CRP: C-reactive protein

Table 4. Frequency of Extrapulmonary Lesions in Patients with and without Rheumatoid Arthritis (RA)

\begin{tabular}{lccc}
\hline & $\begin{array}{c}\text { RA patients } \\
(\mathrm{n}=24)^{*}\end{array}$ & $\begin{array}{c}\text { Non-RA } \\
\text { patients }\end{array}$ & p value \\
\hline Pleuritis and empyema & 2 & 159 & 0.407 \\
Miliary tuberculosis & 2 & 39 & 0.251 \\
Lymph nodes & 1 & 7 & 0.174 \\
Pericarditis & 0 & 7 & 1.000 \\
Intestine & 0 & 5 & 1.000 \\
Vertebrae & 0 & 6 & 1.000 \\
Central nervous system & 0 & 3 & 1.000 \\
(including meningitis) & & & \\
Others & 1 & 4 & 0.113 \\
\hline
\end{tabular}

was changed in $66(6.6 \%)$ of the 995 non-RA patients. The frequency of the need to change the treatment regimen did not differ significantly between the RA patients and non-RA patients.

\section{Antirheumatic treatment and clinical course during hospitalization}

RA symptoms deteriorated during hospitalization in nine patients. One patient developed organizing pneumonia diagnosed via a transbronchial lung biopsy following the initiation of antituberculosis therapy, which was assumed to occur due to worsening of the RA activity. Including this patient, the dose of corticosteroids was increased in eight of the nine patients, with salazosulfapyridine therapy begun in one patient and NSAID therapy started in all nine patients. The RA symptoms then improved in all nine patients. In three other patients, the dose of corticosteroids was increased before the initiation of antituberculosis therapy; the RA symptoms did not worsen in these patients. Ultimately, the antirheumatic treatment received during hospitalization included corticosteroids in 20 patients, MTX in one patient, DMARDs other than MTX in three patients and NSAIDs in
Table 3. Chest X-ray Findings in Patients with and without Rheumatoid Arthritis

\begin{tabular}{lccc}
\hline & $\begin{array}{c}\text { RA patients } \\
(\mathrm{n}=24)^{*}\end{array}$ & $\begin{array}{c}\text { Non-RA patients } \\
(\mathrm{n}=995)\end{array}$ & $\mathrm{p}$ value \\
\hline Laterality & 17 & 602 & $<0.001$ \\
$\quad$ Bilateral & 7 & 374 & \\
Unilateral & 0 & 19 & \\
$\quad$ Unknonw & & 553 & 0.679 \\
Cavity formation & 12 & 438 & \\
$\quad$ Cavitary & 12 & 4 & \\
$\quad$ Non-cavitary & 0 & & \\
$\quad$ Unknown & & &
\end{tabular}

13 of the 24 patients. Five (20.8\%) patients with RA died during hospitalization, in contrast to 149 (15.0\%) patients without RA, while the in-hospital mortality of the RA patients did not differ significantly from that of the non-RA patients. Four of the five RA patient deaths occurring during hospitalization were due to tuberculosis. The other patient developed aspiration pneumonia resulting in empyema and deterioration to death.

\section{Risk factors for in-hospital mortality}

The multivariate analysis revealed an older age, cerebrovascular disease, BMI of $<18.5 \mathrm{~kg} / \mathrm{m}^{2}$, respiratory failure, lymphocytopenia $\left(<700 / \mathrm{mm}^{3}\right)$ and serum albumin level of $<$ $3 \mathrm{~g} / \mathrm{dL}$ to be independent risk factors for in-hospital mortality (Table 5). The presence of bilateral pulmonary lesions, extrapulmonary lesions, congestive heart failure and dementia, the duration from initial symptoms to admission $(\geq 1$ month), an impaired PS, anemia ( $\mathrm{Hb}<11 \mathrm{~g} / \mathrm{dL})$ and the serum total protein level $(<6 \mathrm{~g} / \mathrm{dL})$ were found to be significant risk factors according to the univariate analysis, although they were found to be nonsignificant according to the multivariate analysis. RA itself (odds ratio, 0.509; 95\% CI, 0.144-1.805; $\mathrm{p}=0.296$ ) was not identified to be a significant risk factor in the univariate analysis.

\section{Clinical course after discharge}

Of the 24 patients with RA, 19 were discharged from the hospital. Two patients could not be followed after discharge, as one patient returned to his native country during treatment and the other was found to be missing. The median (range) follow-up time of the remaining 17 patients after discharge was $860(280-3,615)$ days. The antirheumatic drugs taken after discharge by these patients included corticosteroids in 16 cases, MTX in one case, DMARDs other than MTX in one case and NSAIDs in nine cases. The antituberculosis treatment was completed in 12 of these 17 patients. Five patients could not complete the therapy because they died from diseases other than tuberculosis (healthcareassociated pneumonia in one case, aspiration pneumonia in one case, interstitial pneumonia in one case, stroke in one case and senility in one case); however, tuberculosis did not recur during follow-up. Among the remaining 12 patients who completed the treatment, no relapse of tuberculosis oc- 
Table 5. Univariate and Multivariate Analysis of the Risk of In-hospital Mortality in the Study Patients

\begin{tabular}{|c|c|c|c|c|c|c|c|c|c|}
\hline \multirow[b]{2}{*}{ Factor } & \multirow[b]{2}{*}{ Category } & \multirow[b]{2}{*}{$\mathrm{n}$} & \multirow[b]{2}{*}{ Non-survivors } & \multicolumn{3}{|c|}{ Univariate analysis } & \multicolumn{3}{|c|}{ Multivariate analysis (final model) } \\
\hline & & & & OR & $95 \% \mathrm{CI}$ & $\mathrm{p}$ value & OR & $95 \% \mathrm{CI}$ & $\mathrm{p}$ value \\
\hline \multirow[t]{5}{*}{ Age } & $<52$ & 253 & 2 & Reference & - & {$[<0.001]$} & Reference & - & {$[<0.001]$} \\
\hline & $52 \leq,<70$ & 240 & 21 & 12.034 & $(2.790,51.906)$ & $<0.001$ & 7.644 & $(1.622,36.019)$ & 0.010 \\
\hline & $70 \leq,<81$ & 259 & 47 & 27.823 & $(6.679,115.898)$ & $<0.001$ & 13.025 & $(2.851,59.512)$ & $<0.001$ \\
\hline & $81 \leq$ & 264 & 82 & 56.544 & $(13.727,232.906)$ & $<0.001$ & 19.229 & $(4.281,86.361)$ & $<0.001$ \\
\hline & Unknown & 3 & 2 & 250.999 & $(15.656,>999.999)$ & $<0.001$ & 159.448 & $(1.571,>999.999)$ & 0.031 \\
\hline \multirow[t]{2}{*}{ Chest X-ray } & Bilateral & 619 & 131 & 4.512 & $(2.790,7.297)$ & $<0.001$ & & & \\
\hline & Unknown & 26 & 2 & 1.401 & $(0.310,6.329)$ & 0.661 & & & \\
\hline Extrapulmonary lesion & Yes & 215 & 52 & 2.196 & $(1.509,3.194)$ & $<0.001$ & & & \\
\hline \multicolumn{10}{|l|}{ Underlying diseases } \\
\hline CVD & Yes & 99 & 36 & 3.884 & $(2.470,6.108)$ & $<0.001$ & 1.853 & $(1.026,3.348)$ & 0.041 \\
\hline $\mathrm{CHF}$ & Yes & 42 & 14 & 2.989 & $(1.536,5.819)$ & 0.001 & & & \\
\hline Dementia & Yes & 80 & 25 & 2.854 & $(1.717,4.743)$ & $<0.001$ & & & \\
\hline $\mathrm{RA}^{*}$ & Yes & 24 & 5 & 1.495 & $(0.550,4.066)$ & 0.430 & 0.509 & $(0.144,1.805)$ & 0.296 \\
\hline Duration from initial & 1 month $\leq$ & 463 & 64 & 0.626 & $(0.433,0.905)$ & 0.013 & & & \\
\hline symptoms to admission & Unknown & 198 & 17 & 0.367 & $(0.210,0.642)$ & $<0.001$ & & & \\
\hline \multirow[t]{2}{*}{ PS 3,4} & Yes & 266 & 136 & 53.129 & $(29.717,94.988)$ & $<0.001$ & & & \\
\hline & Unknown & 28 & 4 & 8.464 & $(2.592,27.637)$ & $<0.001$ & & & \\
\hline \multirow[t]{2}{*}{ BMI } & $<18.5$ & 279 & 35 & 3.881 & $(2.154,6.993)$ & $<0.001$ & 1.999 & $(1.018,3.926)$ & 0.044 \\
\hline & Unknown & 235 & 101 & 20.391 & $(11.923,34.874)$ & $<0.001$ & 4.572 & $(2.439,8.570)$ & $<0.001$ \\
\hline \multirow[t]{2}{*}{ Respiratory failure } & Yes & 175 & 93 & 15.900 & $(10.562,23.935)$ & $<0.001$ & 4.068 & $(2.489,6.649)$ & $<0.001$ \\
\hline & Unknown & 63 & 9 & 2.337 & $(1.093,4.995)$ & 0.029 & 1.290 & $(0.307,5.415)$ & 0.728 \\
\hline \multirow[t]{2}{*}{ Hemoglobin } & $<11$ & 320 & 82 & 3.244 & $(2.267,4.642)$ & $<0.001$ & & & \\
\hline & Unknown & 22 & 7 & 4.394 & $(1.729,11.169)$ & 0.002 & & & \\
\hline \multirow[t]{2}{*}{ Lymphocytes } & $<700$ & 256 & 100 & 9.886 & $(6.678,14.636)$ & $<0.001$ & 2.688 & $(1.637,4.414)$ & $<0.001$ \\
\hline & Unknown & 24 & 9 & 9.253 & $(3.839,22.302)$ & $<0.001$ & 4.435 & $(0.856,22.975)$ & 0.076 \\
\hline \multirow[t]{2}{*}{ TP } & $<6.0$ & 157 & 74 & 9.192 & $(6.181,13.671)$ & $<0.001$ & & & \\
\hline & Unknown & 59 & 9 & 1.856 & $(0.876,3.930)$ & 0.106 & & & \\
\hline \multirow[t]{2}{*}{ Albumin } & $<3.0$ & 320 & 117 & 33.932 & $(16.272,70.758)$ & $<0.001$ & 7.895 & $(3.565,17.487)$ & $<0.001$ \\
\hline & Unknown & 220 & 29 & 8.939 & $(4.014,19.905)$ & $<0.001$ & 4.451 & $(1.769,11.202)$ & 0.002 \\
\hline
\end{tabular}

Table 6. Characteristics of Patients with RA under Anti-TNF Therapy

\begin{tabular}{|c|c|c|c|c|c|c|c|c|c|c|c|}
\hline Age/Sex & TB & $\begin{array}{c}\text { Underlying } \\
\text { diseases } \\
\text { other than } \\
\text { RA } \\
\end{array}$ & $\begin{array}{l}\text { Duratio } \\
\mathrm{n} \text { of RA }\end{array}$ & $\begin{array}{c}\text { TNF } \\
\text { blockade }\end{array}$ & $\begin{array}{l}\text { Other antirheumatic } \\
\text { treatment on } \\
\text { development of TB }\end{array}$ & $\begin{array}{l}\text { Initial } \\
\text { treatment } \\
\text { of TB }\end{array}$ & $\begin{array}{l}\text { Subsequent } \\
\text { therapy }\end{array}$ & $\begin{array}{c}\text { Outcome of } \\
\text { hospitalization }\end{array}$ & $\begin{array}{l}\text { Readministrati } \\
\text { on of TNF } \\
\text { blockade }\end{array}$ & $\begin{array}{c}\text { Follow-up } \\
\text { period } \\
\text { after } \\
\text { discharge }\end{array}$ & Relapse \\
\hline $68 / \mathrm{F}$ & $\begin{array}{l}\text { Miliary, } \\
\text { pleura }\end{array}$ & $\begin{array}{l}\text { Ssc, } \\
\text { arrhythmia, } \\
\text { HT }\end{array}$ & $10 \mathrm{y}$ & $\mathrm{ADA}$ & $\begin{array}{c}\text { Corticosteroid } 5 \\
\text { mg/day, MTX } 8 \\
\text { mg/week, NSAIDs }\end{array}$ & $\begin{array}{c}\mathrm{INH}+\mathrm{RIF}+ \\
\mathrm{EB}+\mathrm{PZA}\end{array}$ & - & Survived & No & 2 y $10 \mathrm{mo}$ & No \\
\hline $65 / \mathrm{F}$ & $\begin{array}{l}\text { Lung, } \\
\text { pleura }\end{array}$ & HT & $\begin{array}{c}10 \text { y } 11 \\
\text { mo }\end{array}$ & IFX & $\begin{array}{c}\text { MTX } 8 \text { mg/week, } \\
\text { NSAIDs }\end{array}$ & $\begin{array}{c}\mathrm{INH}+\mathrm{RIF}+ \\
\mathrm{EB}+\mathrm{PZA}\end{array}$ & $\begin{array}{l}\text { PZA was } \\
\text { withdrawn } \\
\text { due to liver } \\
\text { dysfunction }\end{array}$ & Survived & No & 1 y $3 \mathrm{mo}$ & No \\
\hline
\end{tabular}

RA: rheumatoid arthritis, TNF: tumor necrosis factor, TB: tuberculosis, HT: hypertension, Ssc: systemic sclerosis, IFX: infliximab, ADA: adalimumab, MTX: methotrexate, NSAIDs: nonsteroidal anti-inflammatory drugs, INH: isoniazid, RIF: rifampicin, EMB: ethambutol, PZA: pyrazinamide, y: years, mo: month

curred during the follow-up period, a median of 1,043 (2803,743 ) days after discharge. Four patients died from causes other than tuberculosis.

\section{RA patients treated with anti-TNF therapy}

The characteristics of the three patients treated with antiTNF therapy are shown in Table 6. TNF blockade was begun with infliximab at two months, adalimumab at three months and infliximab at six months before the development of tuberculosis. Two of the three patients receiving anti-TNF therapy were tested with interferon-gamma release assays (IGRAs) before the administration of biologics; the results were negative in both patients. The results of tuberculin tests in all three patients were negative; thus, none of these patients received treatment for latent tuberculosis infection. Chest X-rays performed prior to the administration of biologics showed no apparent abnormal lesions, although chest computed tomography (CT) performed at the same time revealed a few faint nodules when the images were evaluated retrospectively.

The case of one of the three patients who received antiTNF therapy prior to presentation has been reported previously (14). All three patients treated with anti-TNF therapy developed extrapulmonary tuberculosis. In all three patients, TNF blockade was stopped during treatment for tuberculosis. Corticosteroids and DMARDs other than MTX were 
then administered after discharge, and all three patients completed the antituberculosis therapy. MTX was added in two patients because the RA activity could not be controlled with the current regimen. However, the RA symptoms persisted in one patient, and biologics (etanercept, tocilizumab and abatacept) were administered three years after the completion of tuberculosis treatment. No patients experienced relapse of tuberculosis during the follow-up period.

\section{Discussion}

The characteristics of the RA patients treated without anti-TNF therapy differed from those of the non-RA patients in terms of certain patient demographics, PS, certain laboratory and radiological findings and the frequency of side effects. However, the rate of extrapulmonary lesions and inhospital mortality in the RA patients did not differ significantly from that observed in the non-RA patients.

RA is generally believed to be associated with an increased risk of tuberculosis, which may be attributed to treatment with immunosuppressive agents and the immunological dysfunction caused by the disease itself (6). Although the incidence of tuberculosis has been reported not to increase following conventional immunosuppressive treatment in the United States (15), other studies have suggested that corticosteroids and TNF antagonists (but not MTX) increase the risk of opportunistic infections, including tuberculosis $(16,17)$. Our results showed that RA patients in Japan who develop tuberculosis not uncommonly receive corticosteroids.

To our knowledge, the characteristics of tuberculosis complicating RA were analyzed in only one other study (5), which found that RA patients with tuberculosis are predominantly female. This finding is consistent with our results and may reflect the female predominance observed in RA populations overall. Our finding of fewer RA patients versus non-RA patients with a smoking history may reflect the lower prevalence of smoking among women in Japan in general.

Yoshinaga et al. reported that their RA patients had a higher incidence of bilateral $(70.8 \%)$ and non-cavitary lesions $(64.3 \%)$ than their tuberculosis patients without RA (5). We also found a higher frequency of bilateral lesions in the RA versus non-RA patients in the present study. The radiographic manifestations of pulmonary tuberculosis may be dependent on the level of immunosuppression (18). One reason for the difference in results between the previous report (5) and our study is the differences in patient demographics, including the administered antirheumatic drugs, which affects the level of immunosuppression.

Previous reports have suggested that RA patients treated without anti-TNF therapy have a higher incidence of extrapulmonary tuberculosis (30\%) (5). The frequency of extrapulmonary tuberculosis in our RA patients (33.3\%) did not differ significantly from that observed in the non-RA patients and was similar to that noted in RA patients in the above previous report (5). Therefore, the differences in results between the present and previous study (5) may be due to the differences in the frequency of extrapulmonary tuberculosis among non-RA patients and patient demographics, underlying diseases and administered drugs.

In this study, the RA patients more frequently developed side effects than the non-RA patients. The frequent development of side effects of antituberculosis drugs has not been reported in RA patients; however, in this study, the RA patients were older than the non-RA patients and were assumed to be receiving several drugs, not only antirheumatic drugs, but also drugs for other underlying disorders. Interactions between antituberculosis drugs and other drugs (13) may be one cause of side effects that requires a change in the antituberculosis regimen. Further studies are needed to clarify this issue.

During hospitalization, RA symptoms, including joint pain and swelling worsened in nine patients, all of whom received corticosteroids before admission and RIF after admission. Organizing pneumonia occurred in one of these patients following the administration of antituberculosis treatment, which was assumed to be due to worsening of the RA activity. RIF attenuates the effects of corticosteroids, which was assumed to be the cause of the worsening of RA symptoms in this patient. In such cases, the dose of corticosteroids was increased two to three times above that administered for RA before RIF treatment, which resulted in improvements in the patient's conditions. Patients in whom the corticosteroid dose was increased before the administration of RIF after admission did not experience worsening of RA symptoms. The corticosteroid doses were also increased two to three times above the those administered for RA before admission in these patients. However, the long-term administration of corticosteroids carries the risk of several complications and can result in subsequent or prolonged infection. There have been no reports that DMARDs increase the risk of relapse of tuberculosis; thus, it may be better if other antirheumatic drugs, such as DMARDs, are added to or replace corticosteroids, rather than increasing the dose of corticosteroids. However, which antirheumatic drugs can be used safely and efficiently in such situations has not been fully elucidated. Physicians should be alert to the possibility of worsening of the RA activity during antituberculosis treatment. Further studies are needed to establish adequate strategies for treating RA during antituberculosis treatment.

To our knowledge, the mortality due to tuberculosis in RA patients has not been fully elucidated. In the above mentioned study, the in-hospital mortality rate of the RA patients was similar to that of the non-RA patients (5), as also observed in the present study. In this study, RA itself was not found to be an independent risk factor contributing to in-hospital mortality, whereas several factors previously been reported to be prognostic factors (19-21) were found to be independent prognostic factors. Death from tuberculosis frequently occurs within one to three months after the development of the disease (6) and also occurs in RA patients. Four 
of the five RA patients died of tuberculosis during hospitalization, while no RA patients died from tuberculosis after discharge. Therefore, the management of complications other than tuberculosis is required after discharge.

Twelve of the 19 RA patients who were discharged from our hospital completed a three-month duration of antituberculosis treatment and showed no relapse of tuberculosis during the subsequent follow-up. This suggests that guidelineconcordant therapy can be applied in RA patients receiving immunosuppressive therapy. However, the optimal duration of antituberculosis treatment in patients who develop tuberculosis during antirheumatic drug therapy or due to RA itself remains to be defined. Longer cohort studies with larger populations are needed to determine the duration of antituberculosis treatment that will best reduce relapse rates.

Antirheumatic drugs administered after discharge included corticosteroids in all patients, whereas other drugs were administered in a minority of RA patients. The present study also showed that rheumatologists hesitated to continue or readminister antirheumatic drugs other than corticosteroids that had been administered prior to the diagnosis of tuberculosis during and even after the completion of antituberculosis treatment. In these cases, although the dose of corticosteroids was reduced after discharge in all patients, all patients continued to receive corticosteroids after discharge. Treatment of RA with corticosteroids is widely applied, although a previous report suggested that corticosteroid use results in progressive deterioration of the functional capacity of patients (22). The adverse effects of the long-term use of corticosteroids are well documented and may affect the mortality of RA (23). Although it is not known whether antirheumatic drugs increase the risk of relapse of tuberculosis, the administration of antirheumatic drugs other than corticosteroids may need to be taken into account.

In the present study, three RA patients were treated with TNF antagonists. TNF plays an important role in suppressing mycobacterial infection, and the incidence and relative risk of developing tuberculosis during TNF blockade therapy is reported to be increased $(9,24,25)$. Our three patients received no treatment for latent tuberculosis infection, which decreases the rate of developing tuberculosis in patients at risk of the disease (26). Careful attention should be paid to patients with negative tuberculin skin test and IGRA results, for which the recent TBNET consensus statement suggests that problems exist in their use as screening methods for latent tuberculosis infection (27). Physicians should be alert to not overlook faint radiographic findings. Further discussion is also necessary regarding the use of chest CT to detect faint nodules that may be indicative of tuberculosis and which can only be detected on chest CT.

Our three patients undergoing anti-TNF therapy all developed extrapulmonary tuberculosis, which supports the frequency of extrapulmonary lesions occurring during anti-TNF therapy (1-3). Recently, the frequency of administration of antirheumatic drugs, including anti-TNF therapy, has been altered (28); thus, the characteristics of tuberculosis under antirheumatic treatment may be affected. Longitudinal follow-up of the characteristics of tuberculosis patients must be performed.

In this study, one patient received anti-TNF therapy following the completion of antituberculosis treatment and did not experience relapse of tuberculosis. This finding is compatible with those of a previous report showing that patients completing appropriate antituberculosis treatment do not appear to be at increased risk for tuberculosis, even when antiTNF therapy is started (27).

The present study is associated with several limitations. First, because this study was a retrospective, observational, single-center study, the level of confidence is reduced, and the results may not be applicable in other settings. Second, we did not investigate the disease severity of RA. Although a previous report suggested that the severity of RA is an independent risk factor for infection among RA patients (5), the RA severity at the time of the onset of tuberculosis was noted in only a few of the patient medical records. Therefore, further research of tuberculosis in association with RA and the disease severity of RA is required. Third, the number of patients with RA receiving anti-TNF therapy was limited; thus, no comparisons between RA patients treated with or without anti-TNF therapy were performed.

In conclusion, our results showed that there were minor differences in patient demographics and laboratory findings between the tuberculosis patients with RA treated without anti-TNF therapy and those without RA in this study. The RA patients without anti-TNF therapy exhibited a similar inhospital mortality compared with that of the non-RA patients. All RA patients treated with anti-TNF therapy had extrapulmonary tuberculosis, whereas the rate of extrapulmonary tuberculosis in the other RA patients treated without anti-TNF therapy did not differ from that observed in the non-RA patients. Careful attention should be paid to the adverse effects of antituberculosis drugs and RA activity in RA patients. The present study also suggests that the administration of antirheumatic therapy during and after treatment for tuberculosis should be reconsidered.

\section{The authors state that they have no Conflict of Interest (COI).}

\section{Acknowledgement}

We thank Hideaki Yamakawa, Eriko Kawate, Mina Gochi, Keitaro Nakamoto, Yosuke Miyahara, Yotaro Takaku and Kazuyoshi Kurashima at the Department of Respiratory Medicine, Saitama Cardiovascular and Respiratory Center. We also thank Hideyo Oda of Medical Toukei Co., Ltd. for his valuable advice on the statistical analysis.

\section{References}

1. Takeuchi T, Tatsuki Y, Nogami Y, et al. Postmarketing surveillance of the safety profile of infliximab in 5000 Japanese patients with rheumatoid arthritis. Ann Rheum Dis 67: 189-194, 2008.

2. Koike T, Harigai M, Inokuma S, et al. Postmarketing surveillance of safety and effectiveness of etanercept in Japanese patients with 
rheumatoid arthritis. Mod Rheumatol 21: 343-351, 2011.

3. Dixon WG, Hyrich KL, Watson KD, et al. Drug-specific risk of tuberculosis in patients with rheumatoid arthritis treated with antiTNF therapy: results from the British Society for Rheumatology Biologics Register (BSRBR). Ann Rheum Dis 69: 522-528, 2010.

4. Kaene J, Gershon S, Wise RP, et al. Tuberculosis associated with infliximab, a tumor necrosis factor-alpha-neutralizing agent. $\mathrm{N}$ Engl J Med 345: 1098-1104, 2001.

5. Yoshinaga Y, Kanamori T, Ota Y, et al. Clinical characteristics of Mycobacterium tuberculosis infection among rheumatoid arthritis patients. Mod Rheumatol 14: 143-148, 2004.

6. Yamada T, Nakajima A, Inoue E, et al. Increased risk of tuberculosis in patients with rheumatoid arthritis in Japan. Ann Rheum Dis 65: 1661-1663, 2006.

7. Askling J, Fored CM, Brandt L, et al. Risk and case characteristics of tuberculosis in rheumatoid arthritis associated with tumor necrosis factor antagonists in Sweden. Arthritis Rheum 52: 19861992, 2005.

8. Carmona L, Hernández-García $C$, Vadillo $C$, et al. Increased risk of tuberculosis in patients with rheumatoid arthritis. J Rheumatol 30: 1436-1439, 2003.

9. Gómez-Reino JJ, Carmona L, Valverde VR, et al. Treatment of rheumatoid arthritis with tumor necrosis factor inhibitors may predispose to significant increase in tuberculosis risk: a multicenter active-surveillance report. Arthritis Rheum 48: 2122-2127, 2003.

10. Arnett FC, Edworthy SM, Bloch DA, et al. The American Rheumatism Association 1987 revised criteria for the classification of rheumatoid arthritis. Arthritis Rheum 31: 315-324, 1988.

11. Oken MM, Creech RH, Tomey DC, et al. Toxicity and response criteria of the Eastern Cooperative Oncology Group. Am J Clin Oncol 5: 649-655, 1982.

12. The Japanese Society for Tuberculosis. Guidelines for Clinical Tuberculosis. Nankodo, Tokyo, 2009(in Japanese).

13. Blumberg HM, Burman WJ, Chaisson RE, et al. American Thoracic Society/Centers for Disease Control and Prevention/Infectious Diseases Society of America: treatment of tuberculosis. Am J Respir Crit Care Med 167: 603-662, 2003.

14. Ubukata M, Takayanagi N, Yanagisawa T, et al. Pulmonary tuberculosis with cerebellar lesion in a patient after anti-TNF-alpha (infliximab) treatment for rheumatoid arthritis. Nihon Kokyuki Gakkai Zasshi (Journal of the Japanese Respiratory Society) 43: 247251, 2005 (in Japanese).

15. Wolfe F, Michaud K, Anderson J, et al. Tuberculosis infection in patients with rheumatoid arthritis and the effect of infliximab therapy. Arthritis Rheum 50: 372-379, 2004.
16. Greenberg JD, Reed G, Kremer JM, et al. Association of methotrexate and tumour necrosis factor antagonists with risk of infectious outcomes including opportunistic infections in the CORRONA registry. Ann Rheum Dis 69: 380-386, 2010.

17. Widdifield J, Bernatsky S, Paterson JM, et al. Serious infections in a population-based cohort of 86,039 seniors with rheumatoid arthritis. Arthritis Care Res (Hoboken) 65: 353-361, 2013.

18. Jeong YJ, Lee KS. Pulmonary tuberculosis: Up-to-date imaging and management. AJR Am J Roentgenol 191: 834-844, 2008.

19. Takahara M. Clinical evaluation on causes of death in patients with pulmonary tuberculosis. Kekkaku 79: 711-716, 2004 (in Japanese).

20. Kim DK, Kim HJ, Kwon SY, et al. Nutritional deficit as a negative prognostic factor in patients with miliary tuberculosis. Eur Respir J 32: 1031-1036, 2008.

21. Horita N, Naoki M, Yoshiyama T, et al. Development and validation of a tuberculosis prognostic score for smear positive inpatients: Japanese study. Int J Tuberc Lung Dis 17: 54-60, 2013.

22. Iikuni N, Inoue $\mathrm{E}$, Tanaka $\mathrm{E}$, et al. Low disease activity state with corticosteroid may not represent 'true' low disease activity state in patients with rheumatoid arthritis. Rheumatology (Oxford) 47: 519-521, 2008.

23. Nakajima A, Inoue E, Tanaka E, et al. Mortality and cause of death in Japanese patients with rheumatoid arthritis based on a large observational cohort, IORRA. Scand J Rheumatol 39: 360367, 2010.

24. Keane J, Gershon S, Wise RP, et al. Tuberculosis associated with infliximab, a tumor necrosis factor $\alpha$-neutralizing agent. $\mathrm{N}$ Engl $\mathbf{J}$ Med 345: 1098-1104, 2001.

25. Winthrop KL, Baxter R, Liu L, et al. Mycobacterial diseases and antitumour necrosis factor therapy in USA. Ann Rheum Dis 72: 37-42, 2013.

26. Takeuchi T, Yamanaka H, Inoue E, et al. Retrospective clinical study on the notable efficacy and related factors of infliximab therapy in a rheumatoid arthritis management group in Japan: oneyear outcome of joint destruction (RECONFIRM-2J). Mod Rheumatol 18: 447-454, 2008.

27. Solovic I, Sester M, Gomez-Reino JJ, et al. The risk of tuberculosis related to tumour necrosis factor antagonist therapies: a TBNET consensus statement. Eur Respir J 36: 1185-1206, 2010.

28. Yamanaka H, Inoue E, Singh G, et al. Improvement of disease activity of rheumatoid arthritis patients from 2000 to 2006 in a large observational cohort study IORRA in Japan. Mod Rheumatol 17: 283-289, 2007.

(C) 2014 The Japanese Society of Internal Medicine http://www.naika.or.jp/imonline/index.html 